\title{
Building an Enterprise Data Service at the Federal Reserve Board \\ by San Cannon'
}

\begin{abstract}
Data play a critical role in fulfilling the Federal Reserve Board's mission across a broad range of functions, including monetary policy, financial stability, supervision, consumer protection, and economic research. The current data environment was designed to allow the various business functions to manage relatively small and predictable data sets that required limited sharing across departments or functions, effectively creating business-based silos. The Office of the Chief Data Officer was created in May 2013 to address the data needs of the Board, postfinancial crisis, with an enterprise focus and a clear set of mandates to enhance data governance, data management, and data integration. The OCDO started operations with a small staff of data management professionals who traditionally supported the research function and now must shift gears to provide data services to a broader base of users and a wider range of analytical work. New infrastructures, programs, processes and staffing are being developed and deployed to ensure that data needs across the lifecycle are met and that a variety of analytical approaches can be supported.
\end{abstract}

Keywords: Research, data management, governance, dissemination

\section{Introduction}

Data are the lifeblood of the Board of Governors, and the Federal Reserve System has always been a data-driven organization. Data play a critical role in fulfilling the Board's mission across a broad range of functions, including financial stability, monetary policy, supervision, consumer protection, and economic research. As the Board's mandate has expanded in the wake of the financial crisis and the passage of the Dodd-Frank Act, so has the need for an optimized data environment to meet the breadth and depth of analytical and supervisory challenges the Board is addressing.

The Office of the Chief Data Officer (OCDO) was created in May 2013 through the 2012-2015 Strategic Framework to address major data challenges at the Board, to implement a data governance framework, to improve data management operations cross all divisions and Board-delegated functions, and to strengthen the Board's information sharing environment to optimize the investment in data assets. There are a variety of projects in three strategic areas that must be undertaken to shore up the foundation of our data management operations and position the OCDO to provide the strategic thought leadership around data management and governance for which it was created.

\section{Inventory and metadata}

Critical to the Board's vision for improved data optimization is knowledge of the body of data and content within the Board and across the System. More importantly, we need knowledge not just that data exist, but we need an understanding of their relationship to the organizational mission as well as the internal functions, services, and processes. This includes more complete and better-documented knowledge of information flows, system interconnections, and data security classifications. The overarching vision of the OCDO enterprise data inventory program is to provide the visibility and knowledge of data and their relationship to the people, processes and technology environment in order to support critical data needs and improve data management in a dynamic and 
complex ecosystem. Existing System data-collected, contracted, and created-have not been systemically or strategically cataloged. Moreover, data that have been cataloged have not been cataloged consistently as there are no System-wide standards for doing so. The first stage of the Enterprise Data Inventory (EDI) project was recently completed, identifying necessary metadata elements for capturing relevant information about our data assets. These elements describe physical and logical datasets, roles and responsibilities of the individuals that interact with the data, the business processes and functions that include those data and the applications and technologies where the data reside. Acquisition of a repository to hold these elements and a user interface to make things easy to find are part of the next project steps.

\section{Data management}

Data management is the business function that develops and executes plans, policies, practices, and projects that acquire, control, protect, deliver, and enhance the value of data. The Board's data reside in a number of operational and analytical systems distributed across the Board and System, in varying states of modernization. There are industrial-strength systems that collect and manage data for some departments using time-proven methods and relational database technologies. In other areas, there are quick queries to collect data from financial institutions or other government agencies via spreadsheets that are stored on file shares. Other departments are developing data warehouses and using new "big data" storage technologies. All these repositories meet local and departmental needs but none was developed with the goal of broader access as a key requirement. There has been a pressing increase in the post-crisis needs of the Board to manage a much wider variety of data types across a multitude of data management platforms and to do a better job of sharing those data regardless of their storage location or technology. There have been suggestions in the past that a single enterprise data warehouse would solve all our storage and sharing problems. However, we have determined that there is no one-size-fits-all technology solution to the wide variety and volumes of data that the Board is now responsible for managing. We are engaging in fundamental work will that look at appropriately matching data management technologies with data assets.

The OCDO thinks strategically how to best manage the challenges facing data operations across the Board. As an enterprise service provider, it also needs to build a strong foundation for its own data management operations. The goal is to provide scalable data management services to the Board, and the System where appropriate, that can meet the increasing data capacity demands of the organization. The current staff have historically provided service primarily to the research community at the Board only, but now need to manage data for and disseminate data to other departments with very different business models. The legacy systems for doing the basic intake and output will not scale to support the entire enterprise and a the OCDO has engaged consultants to best determine the technologies and work flow needed for this new level of service is well underway.

\section{Data governance}

Data governance is a core part of the OCDO's mission. Data governance is the exercise of authority, control, and shared decision-making (planning, monitoring, and enforcement) over the management of data assets. Data governance supplies the discipline to deal with both the predictable and unpredictable nature of new data acquisition and data distribution across the organization. Today, data governance activities occur in business lines with little or no formal coordination across the Board and across Board-delegated functions. Little of this work is automated, and even then generally not beyond the use of SharePoint sites as document repositories.

The OCDO will facilitate a variety of data governance activities through its Data Governance Program (DGP). The DGP serves as a conduit for coordination, communication, and decision-making across and within business lines and departments within the Board. The DGP has responsibility for developing the body of policies, processes, standards, and metrics; communicating progress and informing the organization about ongoing and completed activities; and, completing specific DGP actions, deliverables and activities.

Once foundational projects in these functional areas are underway, the Board will be better positioned to undertake more strategic work to provide discovery services. To improve the Board's ability to find, layer, and explore a wide variety of data in unique ways is an important goal to better serve the Board's mission critical work which is more multi-disciplinary and cross-functional than it has ever been. We are planning work that supports the notion of discovery through the development of an enterprise-wide information architecture that shows the conceptual and logical relationships between different types of data regardless of the technology or application that supports the data. This includes the development of subject area models and taxonomies as well as the development of analytics, visualizations and knowledge management services. Some of this has been done at the departmental level in the past but the OCDO focus is to provide such services for data users across the Board.

Additional services

Other services will be needed to support the growing scope of work for an enterprise data service. The creation of a formal information architecture practice will lay the foundation for more complete data modeling and documentation of the logical and conceptual relationships between data assets. Business analysts will help map business processes and evaluate workflow efficiencies. Formal change management and communication will help staff to better understand what the OCDO can do for them, rather than worrying about what the Office is trying to do to them. Project management and training complete the service portfolio and will help keep work moving forward and users better informed about what data we have and how it can or should be used.

\section{Conclusion}

While the Federal Reserve Board is midway through the strategic plan that brought centralized data management and governance into being, the work will extend far beyond this strategic plan and will likely be an important theme in the next plan. When fully staffed at just under 50 staff members, the Office of the Chief Data Officer will have to be efficient, effective, collaborative and communicative to be able to serve as an enterprise service and work effectively in an institution with long standing business line traditions and work flows.

\section{NOTES}

1.San Cannon, Deputy Chief Data Officer, Federal Reserve Board, Washington, D.C. 20551 sandra.a.cannon@frb.gov 